\title{
Retrieval of canopy component temperatures through Bayesian inversion of directional thermal measurements
}

\author{
J. Timmermans ${ }^{1}$, W. Verhoef ${ }^{1,2}$, C. van der Tol ${ }^{1}$, and Z. Su${ }^{1}$ \\ ${ }^{1}$ International Institute for Geo-Information Sciences and Earth Observation (ITC), Hengelosestraat 99, P. O. Box 6, \\ 7500 AA, Enschede, The Netherlands \\ ${ }^{2}$ National Aerospace Laboratory (NLR), Anthony Fokkerweg 2, 1059 CM, Amsterdam, The Netherlands
}

Received: 29 January 2009 - Published in Hydrol. Earth Syst. Sci. Discuss.: 2 April 2009

Revised: 12 June 2009 - Accepted: 29 June 2009 - Published: 21 July 2009

\begin{abstract}
Evapotranspiration is usually estimated in remote sensing from single temperature value representing both soil and vegetation. This surface temperature is an aggregate over multiple canopy components. The temperature of the individual components can differ significantly, introducing errors in the evapotranspiration estimations. The temperature aggregate has a high level of directionality. An inversion method is presented in this paper to retrieve four canopy component temperatures from directional brightness temperatures. The Bayesian method uses both a priori information and sensor characteristics to solve the ill-posed inversion problem. The method is tested using two case studies: 1) a sensitivity analysis, using a large forward simulated dataset, and 2) in a reality study, using two datasets of two field campaigns. The results of the sensitivity analysis show that the Bayesian approach is able to retrieve the four component temperatures from directional brightness temperatures with good success rates using multi-directional sensors $\left(S_{r}^{\text {spectra }} \approx 0.3, S_{r}^{\text {gonio }} \approx 0.3\right.$, and $S_{r}^{\mathrm{AATSR}} \approx 0.5$ ), and no improvement using monoangular sensors $\left(S_{r} \approx 1\right)$. The results of the experimental study show that the approach gives good results for high LAI values $\left(\mathrm{RMSE}_{\text {grass }}=0.50 \mathrm{~K}, \quad \mathrm{RMSE}_{\text {wheat }}=0.29 \mathrm{~K}\right.$, $\left.\mathrm{RMSE}_{\text {sugar beet }}=0.75 \mathrm{~K}, \quad \mathrm{RMSE}_{\text {barley }}=0.67 \mathrm{~K}\right) ; \quad$ but for low LAI values the results were unsatisfactory $\left(\mathrm{RMSE}_{\text {young maize }}=2.85 \mathrm{~K}\right)$. This discrepancy was found to originate from the presence of the metallic construction of the setup. As these disturbances, were only present for two
\end{abstract}

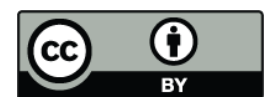

Correspondence to: J. Timmermans (timmermans@itc.nl) crops and were not present in the sensitivity analysis, which had a low LAI, it is concluded that using masked thermal images will eliminate this discrepancy.

\section{Introduction}

The retrieval of canopy component temperatures is of great importance for the calculation of evapotranspiration by remote sensing. Evapotranspiration in remote sensing is usually calculated by applying energy balance models like SEBAL (Bastiaanssen et al., 1998), and SEBS (Su, 2002). Such algorithms are highly sensitive to errors in air temperatures, the retrieved kinematic temperatures and emissivities.

In most remote sensing energy balance studies only a single surface temperature is used ( $\mathrm{Su}, 2002$; Jia et al., 2003a). This surface temperature is calculated from the emitted radiances using Planck's radiance function, with emissivities estimated a priori or calculated using techniques like temperature emissivity separation (Payan and Royer, 2004 and Gillespie et al., 1998). This method is widely used and produces good results. It can however only retrieve a single surface temperature.

For heterogeneous canopies, the assumption of a single surface temperature is not correct. Timmermans et al. (2008) showed that in this case the temperatures of the individual canopy components will differ significantly. Thermal remote sensing sensors, like ASTER usually have a spatial resolution of more than $60 \mathrm{~m}$ (Yamaguchi et al., 1998). The measured radiation by these sensors will be an aggregation of radiation emitted by the different canopy components. This aggregation generates a high level of directionality. For example, as

Published by Copernicus Publications on behalf of the European Geosciences Union. 
the optical path through the canopy is large for more oblique viewing angles, the thermal brightness temperature will depend more on vegetation than on soil for these angles (Verhoef et al., 2007a). The level of directionality further depends on several parameters like the temperature of the individual canopy component and LAI.

Directional remote sensing is therefore the best tool for the retrieval of canopy component temperatures. Jia et al. (2003b) showed that the retrieval of multiple independent temperatures requires multiple observations to avoid an indeterminate system.

Most of the parameter retrieval studies deal with the optical part of the spectrum, illustrated by D'Urso et al. (2004) and Knyazikhin et al. (1998). Only a few studies have investigated the retrieval of kinematic component temperatures (Kustas and Norman, 2000; Yan et al., 2001; Jia et al., 2003b). These studies limit the number of retrieved canopy component temperatures to two, namely those of soil and canopy.

The objective of this research was to create an inversion scheme to retrieve four component temperatures. This inversion scheme was tested on the forward simulated brightness temperatures. A sensitivity analysis was performed with various satellite configurations, from single view and single band to multiple view and multiple bands. Finally a case study was performed using data from two field campaigns: EAGLE 2006 and AGRISAR 2006. The directional brightness temperatures that are used were measured using various instruments attached to a goniometer.

\section{Methodology}

Biophysical parameters can be retrieved with high accuracy using radiative transfer models (Gastellu-Etchegorry et al., 2003). The success rate and number of parameters that can be retrieved depends on 1) the inversion method, 2) the number of independent observables and 3) the sensitivity of the modelled remotely sensed data to surface properties.

The inversion algorithm has been implemented into a Matlab program. This program has been run on a multi-processor system. The data flow of this program is shown in Fig. 1. The different aspects of the inversion, like the algorithm and the radiative transfer model are, discussed in the following paragraphs.

\subsection{Traditional retrieval algorithms}

According to Kimes et al. (2000) retrieval algorithms can be divided into three major classes: neural network, lookup tables and optimization schemes. The accuracy of neural networks and look-up tables depends on the number of precomputed "training" values and on the interpolation between these "trained" values (Picton, 2000). This interpolation can not be performed over singularities like the hot spot, despite

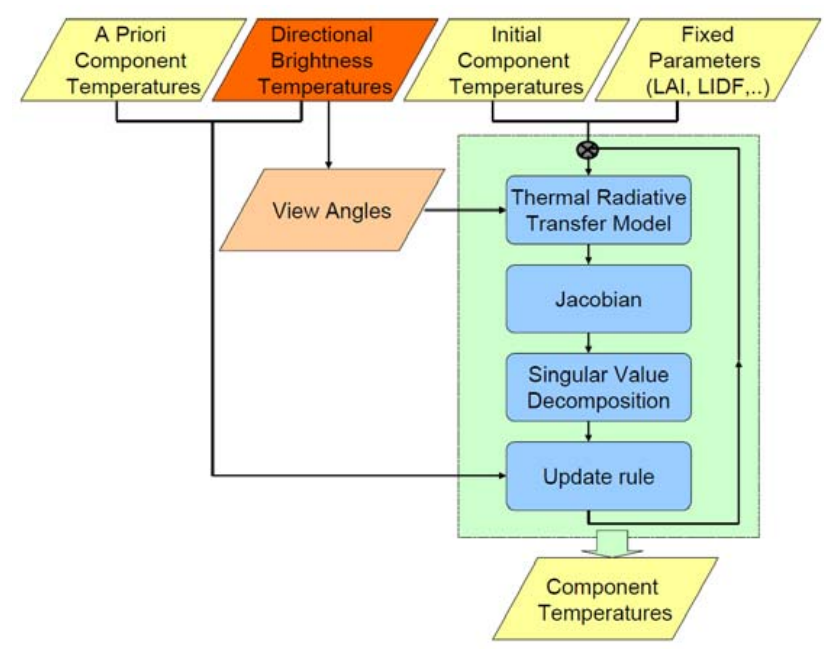

Fig. 1. Bayesian Inversion Scheme.

recent advances have been made for look-up table methods by Gastellu-Etchegorry et al. (2003). Optimization methods do not have this limitation, because of the radiative transfer model that is used. Optimization methods are inherently suitable to handle arbitrary viewing and observing angles. Therefore the optimization method is still the most used inversion technique in directional remote sensing.

A simple optimization update is based on the GaussNewton method (Tarantola, 2005), see Eq. (1).

$\Delta \boldsymbol{p}=\left(\mathbf{J}^{T} \mathbf{J}\right)^{-1} \mathbf{J}^{T} \Delta \boldsymbol{r}$

Here $\boldsymbol{\Delta} \boldsymbol{p}$ denotes the changes in the parameters for retrieving the correct parameter set, $\Delta \boldsymbol{r}$ denotes the difference between the observed variables and simulated variables, and $\mathbf{J}$ denotes the local sensitivity of the model.

Newton methods are relatively easy to implement and converge fast (quadratically). To improve upon this convergence the Newton method was modified independently by Levenberg and Marquardt (Fox, 1997). The Levenberg-Marquardt (LM) algorithm is a trust-region modification of the GaussNewton algorithm (Moré and Wright, 2003).

$$
\boldsymbol{\Delta} \boldsymbol{p}=\left(\mathbf{J}^{T} \mathbf{J}+\mu \mathbf{I}\right)^{-1} \mathbf{J}^{T} \boldsymbol{\Delta} \boldsymbol{r}
$$

here $\mu$ is a scalar that controls the numerical stability of the algorithm, see Verhoef (2000). This (non-negative) scalar is updated per iteration. When $\mu$ is small the LM method approaches the quadratic Newton convergence; when $\mu$ is large the LM method approaches the steepest descent method. This LM method is still used in current researches (North, 2002). Both methods however still lead to instability as can be shown using singular value decomposition.

If the input parameters of the model are linearly dependent on each other, the input-output relationship will be nonunique. This results in an ill-posed inversion scheme, making it impossible to retrieve unique sets of parameters. Single Value Decomposition (SVD) will not only diagnose this 
problem but will give you a useful numerical answer (Press et al., 2007). The SVD method states that a Jacobian matrix $J$ can be decomposed as

$\mathbf{J}=\mathbf{U S V}^{T}$

with $\mathbf{U}$ column-orthogonal $\left(\mathbf{U}^{T} \mathbf{U}=\mathbf{I}\right), \mathbf{S}$ diagonal and $\mathbf{V}$ orthogonal $\left(\mathbf{V}^{T} \mathbf{V}=\mathbf{V} \mathbf{V}^{T}=\mathbf{I}\right)$. This results in the relation $\left(\mathbf{J}^{T} \mathbf{J}\right) \mathbf{J}^{T}=\mathbf{V S}^{-1} \mathbf{U}^{T}$, which illustrates why the GaussNewton method may be suffering from difficulties, since the matrix of singular values $S$ may contain zeros. This happens when there is linear dependence, and in that case the matrix inversion is impossible. For small singular values the corresponding change of model input parameters becomes very large, which leads to numerical instability.

\subsection{Bayesian optimization algorithm}

Kimes (2000) and Verhoef (2007b) showed that the ill-posed problem of model inversion can be reduced if a priori data is used. The addition of a priori information transforms the original Newtonian update rule, Eq. (1), into Eq. (4).

$\boldsymbol{\Delta} \boldsymbol{p}=\mathbf{V}\left(\mathbf{S}^{2}+\mathbf{I}\right)^{-1}\left[\mathbf{S} \mathbf{U}^{T} \boldsymbol{\Delta} \boldsymbol{r}+\mathbf{V}^{T} \boldsymbol{\Delta} \boldsymbol{p}_{\boldsymbol{a}}\right]$

Here $\Delta \boldsymbol{p}_{\boldsymbol{a}}$ denotes the difference between the current parameter set, $\boldsymbol{p}_{\boldsymbol{m}}$, and the a priori values, $\boldsymbol{p}_{\boldsymbol{a}}$, and $\Delta \boldsymbol{r}_{\boldsymbol{m}}$ denotes the difference between the measurements, $\boldsymbol{r}$, and modelled observations, $\boldsymbol{r}_{\boldsymbol{m}}$. The variables are normalized by the respective standard deviations of the parameters, assuming a Gaussian distribution. The variable $\boldsymbol{\Delta} \boldsymbol{r}$ is normalized using the sensor accuracy.

The final solution of the inversion is then given by Eq. (5). It can be seen that when the sensitivity of the model is high the model solution will dominate the solution and when it is low, the a priori solution will.

$\boldsymbol{p}_{\boldsymbol{s}}=\mathbf{V}\left(\mathbf{S}^{2}+\mathbf{I}\right)^{-1}\left[\mathbf{S}^{2} \mathbf{V}^{T} \boldsymbol{p}_{\boldsymbol{m}}+\mathbf{V}^{T} \boldsymbol{p}_{\boldsymbol{a}}\right]$

A priori information on the temperature is extracted from the shape of the directional thermal brightness temperature. The path length through the canopy depends on the viewing angle; for oblique viewing angles $\theta_{o}$, the optical path length will be large and the measured brightness temperature will depend mainly on the vegetation itself. $T_{v}=T_{b}\left(\theta_{o}=\max \left(\theta_{o}\right)\right)$, and for nadir looking angles, the optical path length is at a minimum and the measured brightness temperature will depend more on the soil. $T_{v}=T_{b}\left(\max \left(\theta_{o}\right)\right)$.

The standard deviation used to normalize this a priori information is calculated using the upper and lower limit for the vegetation and soil, assuming a Gaussian distribution. The lower and upper limits for soil are set to $-5^{\circ} \mathrm{C}$ and $100^{\circ} \mathrm{C}$, and for vegetation $0^{\circ} \mathrm{C}$ and $42^{\circ} \mathrm{C}$.

\subsection{Sensitivity and uncertainty}

This Bayesian update rule not only solves the ill-posedness of the inversion problem, but also takes into account the sensitivity of the model and uncertainties in input/output parameters. The sensitivity of the radiative transfer model to the input parameters is captured by the diagonal matrix $\mathrm{S}$. The uncertainty of the model and measurements is captured by the normalization of $\Delta \boldsymbol{r}$ and $\Delta \boldsymbol{p}_{\boldsymbol{a}}$. For example, if the sensitivity is low, the values in $S$ will become nearly singular. In that case the update rule will be largely dependent on the a priori values.

\subsection{Radiative transfer models}

The radiative part of the Soil Canopy Observation, Photochemistry and Energy fluxes (SCOPE) model (Tol et al., 2009) was used in the inversion. SCOPE is a soil-vegetationatmosphere transfer model. It calculates the biophysical and spectral fluxes for individual leaves. It scales these fluxes up to canopy level. SCOPE uses existing models of radiative transfer, micrometeorology and plant physiology. It uses a numerical version of the 4SAIL model.

The original SAIL model (Verhoef, 1984) is a onedimensional directional radiative transfer model. SAIL has been used in many studies. It also has been validated several times by studies like the RAMI comparison (Pinty et al., 2001; Pinty et al., 2004; Widlowski et al. 2007). Recently, Verhoef et al. (2007a) have expanded the earlier versions of the model in order to simulate also thermal radiative transfer inside a canopy, see Eq. (6). As the optical part of the spectrum can be solved analytically, this part of the model is very efficient both in computer resources as in physical representation. The calculation of the directional thermal brightness temperature increases computation time only slightly. This makes the SAIL model ideal for inversion of thermal brightness temperatures.

$$
\begin{aligned}
E_{o}= & r_{s o}^{*} E_{s}+r_{d o}^{*} E^{-}+\varepsilon_{v}^{*} H_{c}+\varepsilon_{s}^{*} H_{d}+\varepsilon_{v}^{* *} \\
& \left(H_{h}-H_{c}\right)+\varepsilon_{s}^{* *}\left(H_{s}-H_{d}\right)
\end{aligned}
$$

Here, $E_{o}$ is the directional radiation, $E_{s}$ the downwelling solar radiation, $E^{-}$the downwelling diffuse radiation, $H_{h}$ and $H_{c}$ are the hemispherical thermal radiation fluxes for sunlit (hot) and shaded (cold) leaves, $H_{s}$ and $H_{d}$ are the hemispherical thermal radiation fluxes for sunlit and shaded soil. The parameter $r_{s o}^{*}$ is the total reflectance for direct radiation, $r_{d o}^{*}$ is the total reflectance for diffuse radiation, $\varepsilon_{v}^{*}$ and $\varepsilon_{v}^{* *}$ are the effective emissivities for sunlit and shaded vegetation, and $\varepsilon_{s}^{*}$ and $\varepsilon_{s}^{* *}$ are the effective emissivities for sunlit and shaded soil. These parameters are calculated by SAIL using single leaf thermal emissions and single leaf reflectance/transmittance spectra (Table 1). 
Table 1. SAIL required Input Parameters. The LAI value given is only used in the sensitivity analysis, as LAI was measured during the field campaigns.

\begin{tabular}{|c|c|c|c|}
\hline LAI & Leaf Area Index & 1.50 & \\
\hline $\mathrm{h}$ & Hotspot parameter & 0.05 & \\
\hline $\operatorname{LIDF}_{a}$ & Leaf Inclination density function parameter a & -0.35 & \\
\hline $\mathrm{LIDF}_{b}$ & Leaf Inclination density function parameter $b$ & -0.15 & \\
\hline$\rho_{l}$ & Single leaf reflectance & PROSPECT & \\
\hline$\varepsilon_{v}^{*}$ & Single leaf emissivity (sunlit) & 0.99 & \\
\hline$\varepsilon_{v}^{* *}$ & Single leaf emissivity (shaded) & 0.99 & \\
\hline$\theta_{s}$ & Sun zenith angle & [0-60] & $\circ$ \\
\hline$\theta_{s}$ & Observer zenith angle & sensor dependant & $\circ$ \\
\hline$\psi$ & Relative sun-object azimuth angle & sensor dependant & $\circ$ \\
\hline
\end{tabular}

Table 2. Input parameters for single leaf reflectance and emission.

\begin{tabular}{llll}
\hline $\mathrm{N}$ & Mesophyll structure parameter & 1.400 & \\
\hline $\mathrm{C}_{a b}$ & Chlorophyll a and b content & 60.000 & $\mu \mathrm{g} / \mathrm{cm}^{2}$ \\
$C_{w}$ & Water Content & 0.009 & $\mathrm{~cm}$ \\
$C_{m}$ & Dry matter & 0.012 & $\mathrm{~g} / \mathrm{cm}^{2}$ \\
$C_{s}$ & Senescent material & 0.000 & \\
$T_{c}$ & Sunlit Leaf Temperature & See Fig. 2. & $\left({ }^{\circ} \mathrm{C}\right)$ \\
$T_{c}$ & Shaded Leaf Temperature & See Fig. 2. & $\left({ }^{\circ} \mathrm{C}\right)$ \\
\hline
\end{tabular}

Single leaf reflectance is calculated by the PROSPECT model (Jacquemoud et al., 1990). This model is able to parameterize the leaf reflectance using 5 parameters. These parameters are listed in Table 2.

Emitted single leaf thermal radiation is calculated using Planck's radiance function on basis of the component temperatures (Table 2). Planck's equation, Eq. (7), calculates the emitted flux per wavelength and bandwidth (Quattrochi and Luvall, 2004):

$H(\lambda)=\pi I(\lambda)=2 \pi \int_{\lambda_{1}}^{\lambda_{2}} \frac{h c^{2}}{\lambda^{5} \exp [(h c / \lambda k T)-1]} d \lambda$

here $\lambda$ is the wavelength (m), $h$ denotes the Planck constant (Js), $c$ the speed of light, $k$ Boltzmann's constant $\left(\mathrm{J} \mathrm{K}^{-1}\right)$ and $T$ the temperature of the component $(\mathrm{K})$. The integration is performed over bandwidth $\lambda_{2}-\lambda_{1}$.

The soil reflectance is not modelled but a standard spectrum, gravelly soil, is taken from the DART spectral database (Gastellu-Etchegorry et al., 2004) and Lambertian reflectance is assumed. The soil thermal emission are calculated using the Eq. (7), but with the sunlit and shaded contact temperatures, $T_{h}$ and $T_{d}$.

Note that the current method assumes that the component temperatures are homogeneous per component. However the temperature of sunlit leaves sunlit leaves has variations in the
Table 3. Input parameters for soil reflectance and emission.

\begin{tabular}{llll}
\hline & & Gravelly & \\
$\rho_{s}$ & DART spectral database () & Soil & \\
\hline$\varepsilon_{s}^{*}$ & Soil emissivity (sunlit) & 0.95 & \\
$\varepsilon_{s}^{* *}$ & Soil emissivity (shaded) & 0.95 & \\
$T_{h}$ & Sunlit Soil Temperature $\left({ }^{\circ} \mathrm{C}\right)($ hot $)$ & See Fig. 2. & $\left({ }^{\circ} \mathrm{C}\right)$ \\
$T_{c}$ & Shaded Soil Temperature $\left({ }^{\circ} \mathrm{C}\right)($ cold) & See Fig. 2. & $\left({ }^{\circ} \mathrm{C}\right)$ \\
\hline
\end{tabular}

range of $5-10 \mathrm{~K}$ around the average temperature. This variation in temperature depends on the biophysical processes in the leaf. As the inversion method will only be run over the radiative transfer part, these biophysical processes will not be taken into account, and we will retrieve the four "mean" component temperatures. The inversion method can also be used over the complete SCOPE model, but due to computational demands this is left out of this paper.

\section{Case study}

The algorithm is test using both simulated and measured data of directional brightness temperatures. Concurrently to the acquisition of the directional brightness temperature in the field, measurements on vegetation structure and emissivity were performed.

In time-limited field campaigns the chance of encountering all these scenarios needed for a thorough sensitivity analysis is very small. In order to explore the performance of the algorithm in different conditions, forward simulated brightness temperatures are used. The advantage of forward simulations is that one can also simulate future satellites like SPECTRA (Sobrino and Jiménez-Munõz, 2005; Verhoef, 2007a). 
Table 4. Sensor characteristics. ${ }^{1}$ The number of thermal bands shown here is the amount of bands with wavelength $>8 \mu \mathrm{m} .{ }^{2}$ The Irisys 1011 is attached to a goniometric system, enabling complete hemispherical coverage; the number of observations angles is divided over 1 nadir, 4 across-solar-plane 4 across-solar-plane.

\begin{tabular}{lrrrrr}
\hline & $\begin{array}{r}\text { Nr. } \\
\text { Thermal } \\
\text { Bands }\end{array}$ & $\begin{array}{r}\text { Thermal } \\
\text { Bands } \\
\text { Spectrum }\end{array}$ & $\begin{array}{r}\text { Thermal } \\
\text { Spectral } \\
\text { Resolution }\end{array}$ & $\begin{array}{r}\text { Absolute } \\
\text { Thermal } \\
\text { Accuracy }\end{array}$ & $\begin{array}{r}\text { Nr. } \\
\text { Observation } \\
\text { Angles }\end{array}$ \\
\hline Irisys 1011 & & $(\mu \mathrm{m})$ & $(\mu \mathrm{m})$ & $(\mathrm{K})$ & \\
Landsat 7 & 1 & $08.00-14.00$ & 6.00 & 0.5 & 1 (nadir) \\
AATSR & 1 & $10.40-12.50$ & 2.10 & 0.6 & 1 (nadir) \\
ASTER & 2 & $10.40-12.50$ & $0.90-1.00$ & 0.1 & 1 (nadir) \\
MODIS & 5 & $08.12-11.65$ & $0.35-0.70$ & 1.0 & 1 (nadir) \\
AATSR & 5 & $08.40-14.39$ & 0.50 & 0.5 & 1 (nadir) \\
SPECTRA & 2 & $10.40-12.50$ & $0.90-1.00$ & 0.1 & 2 \\
Irisys 1011 (Goniometer) & 2 & $10.30-12.80$ & 0.50 & 1.0 & 7 \\
\hline
\end{tabular}

\subsection{Sensitivity analysis}

The forward simulations were performed for different sensors and for different scenarios. The total number of simulations was 3840 scenarios (consisting of 8 sensors, 8 component temperature profiles, 10 solar Zenith angles and 6 noise profiles. Note that in reality the component temperature profiles are the result of the surface energy balance and consequently the solar zenith angles. However in this case the temperatures are set independently for the sensitivity analysis.

\subsubsection{Number of parameters}

The retrieval algorithm was executed over each of these scenarios three times. The number of parameters to be retrieved was varied during these retrievals, to investigate how close the inversion was to becoming ill-posed. These extra parameters that will be retrieved are the air temperature and the surface temperature. These parameters were proposed due to their high significance in estimating evapotranspiration and the difficulty in current retrieving algorithms.

\subsubsection{Sensors}

In order to investigate the potential of multidirectional remote sensing several sensors have been simulated, see Table 2. These instruments range from broadband ground instrument, like the Irisys 1011 Thermal Camera (Sobrino et al., 2005) to multispectral solo-directional satellite sensors, like ASTER (Yamaguchi et al., 1998) and multispectral multidirectional satellite sensors, like AATSR (Prata et al., 1990).

\subsubsection{Temperature profiles}

The number of parameters that can be retrieved depend mainly on the number of independent angular measurements. The level of directionality depends mainly on the differences in temperatures of the individual components. The lower the difference in component temperatures, the lower the level of directionality in the brightness temperatures is. This limits the number of independent angular measurements ( $\mathrm{Li}$ et al., 2003).

Therefore different component temperature profiles were used to investigate the limitations of the retrieval algorithm. In total 8 profiles were simulated, representing summer, winter and autumn/spring profiles and 1 homogeneous profile (Fig. 2).

\subsubsection{Solar angles}

The shape of the directional brightness temperature depends greatly on the solar angle. The solar angle controls the sunlit fraction of soil and vegetation. Differences in irradiance cause heterogeneity in temperatures through energy balance. A thermal hot spot may be observed when sunlit soil is warmer than shaded soil.

\subsubsection{Noise}

The directional behaviour of the brightness temperatures can be lost if the noise in the measurement is too high. Therefore the effect of different levels of noise on the retrieval algorithm should be investigated. Different levels of sensor noise were added to the directional brightness temperatures in order to investigate the success of retrieval algorithm as is shown in Eq. (8). Regarding the SAIL-inherent error, this was considered to be outside the scope of this present paper. 
A

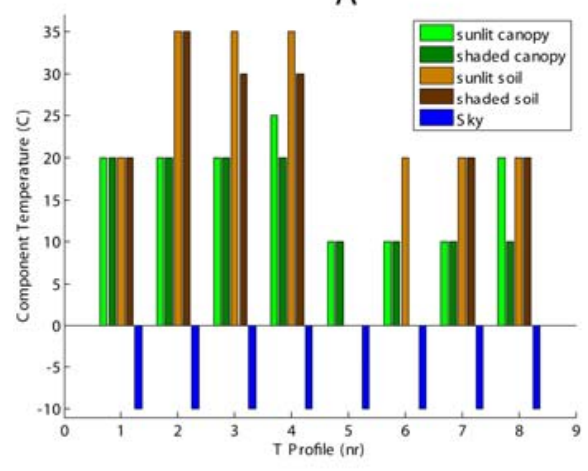

B

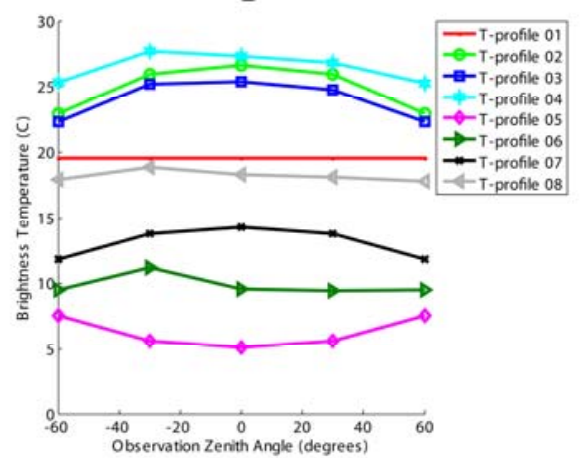

Fig. 2. Temperature Profiles. In (A) the temperature profiles. In (B) the corresponding directional brightness temperatures are shown. The a-symmetry in the directional brightness temperature is caused by the difference in observed sunlit/shaded vegetation/soil.

$T_{\mathrm{bm}}\left(i_{\text {band }}, i_{\text {obs }}\right)=T_{\text {bt }}\left(i_{\text {band }}, i_{\text {obs }}\right)+d T_{\text {bn }}\left(i_{\text {band }}, i_{\text {obs }}\right)$

with

$d T_{\mathrm{bn}}\left(i_{\mathrm{band}}, i_{\mathrm{obs}}\right)=N_{l} A_{s}\left(i_{\mathrm{band}}\right) \Re\left(i_{\mathrm{band}}, i_{\mathrm{obs}}\right)$

The measured brightness temperature, $T_{\mathrm{bm}}$ at band $i_{\text {band }}$ and observation angle $i_{\mathrm{obs}}$, is calculated as the sum of the "true" brightness temperature, $T_{\mathrm{bt}}$ and the noise $d T_{\mathrm{bn}}$. The noise is calculated as the product of a noise level $N_{l}$, the sensor accuracy $A_{s}$ and random value, $\Re$. The random values are generated with a normal distribution $($ std $(\Re)=1$ and mean $(\Re)=0)$.

Note that most researches simulate sensor noise by using the specified noise equivalent temperature difference (NEdT) that is given in the sensor documentations. This study aims to investigate the success rate of the algorithm for other than sensor noise as well. For this reason, the NEdT approach is considered too limited for this study.

\subsubsection{Success rate}

The success of the retrieval is evaluated using the ratio of $\mathrm{RMSE}_{m}$ and $\mathrm{RMSE}_{i}$, see Eq. (10).

$S_{r}=\frac{\operatorname{RMSE}_{m}}{\operatorname{RMSE}_{i}}$

With

$\operatorname{RMSE}_{m}=\sqrt{\frac{1}{N} \sum_{i}^{N}\left(\boldsymbol{p}_{\boldsymbol{m}}(i)-\boldsymbol{p}_{\boldsymbol{t}}(i)\right)^{2}}$

$\mathrm{RMSE}_{i}=\sqrt{\frac{1}{N} \sum_{i}^{N}\left(\boldsymbol{p}_{\boldsymbol{a}}(i)-\boldsymbol{p}_{\boldsymbol{t}}(i)\right)^{2}}$

Here, $S_{r}$ is the success rate, $\mathrm{RMSE}_{m}$ is the root mean square error between the retrieved parameters, $\boldsymbol{p}_{\boldsymbol{m}}$, and true parameters, $\boldsymbol{p}_{\boldsymbol{t}}$; $\mathrm{RMSE}_{i}$ is the root mean square error between the a priori parameters, $\boldsymbol{p}_{\boldsymbol{a}}$, and true parameters. Values for $S_{r}$ lower than 1 denote that the inversion has improved compared to the a priori estimates of the parameters; values for $S_{r}$ higher than 1 denote that the inversion has deteriorated the a priori estimates; values of 1 denote that the inversion has neither improved or deteriorated upon the a priori estimates.

Note that the success rate uses averages the separate error for the different parameters. As the algorithm is based on the Gauss-Newton method, it optimizes the overall error resulting in larger errors for components that are less frequent (such as sunlit soil in the case of high LAI).

\subsection{Field measurements}

The field measurements were acquired with an Irisys 1010 thermal radiometer (Sobrino et al., 2005) that was attached to a goniometer (Timmermans et al., 2009; Li et al., 2004). A goniometer is a device enabling complete hemispherical measurements. The total number of angular positions per run was 9. After each run a complimentary run was performed at 45 degrees offset in the azimuthal direction. The combination of the two runs takes $5 \mathrm{~min}$. The meteorological parameters and nadir brightness temperatures were observed in order to characterize the dynamical change in component temperatures. In the post processing the runs were filtered based on these observations.

The datasets that are used were acquired in two field campaigns. The first dataset contains the field measurements of the EAGLE 2006 field campaign (Su et al., 2007, 2009). This field campaign was organized by the ITC in collaboration with the ESA in the EAGLE framework. The measurements were performed over young maize $(\mathrm{LAI}=0.2)$ and tall grass (LAI=2.5). The sites are part of the KNMI measurement facility situated near the Cabauw meteorological tower (Van Ulden and Wieringa, 1996), in the central part of the Netherlands. The second dataset was acquired during the AGRISAR 2006 field campaign. The measurements were 
A

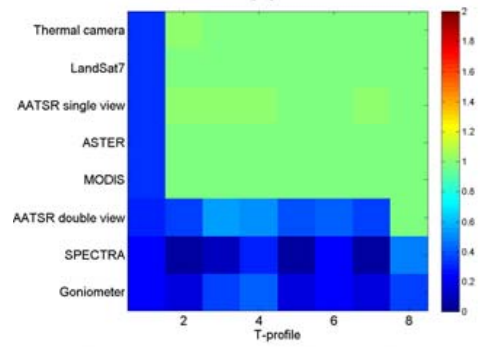

C

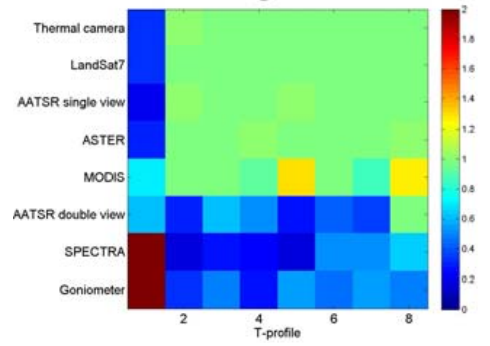

B

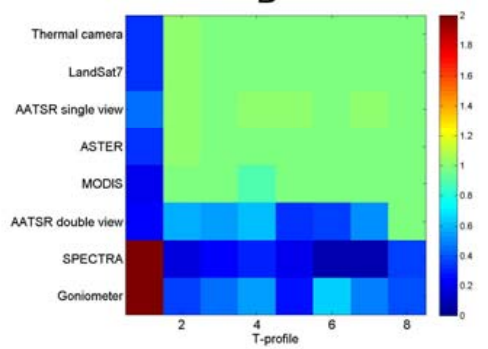

D

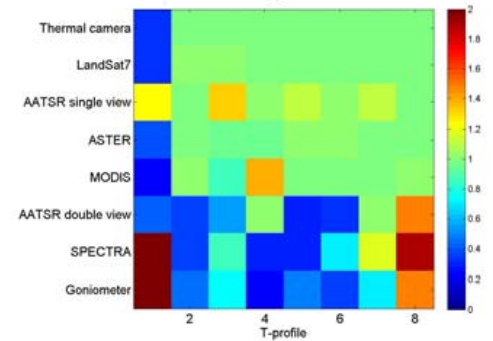

Fig. 3. Success rate of different satellites for different temperatures and noise levels. The solar zenith angle for these simulations was 46.7 degrees. (A-D) show respectively a noise level of 0.0, 0.4, 0.8 and 1.0.

performed over mature maize ( $\mathrm{LAI}=1.5)$, wheat $\mathrm{LAI}=4.0$ ), sugar beet $(\mathrm{LAI}=2.0)$ and barley $(\mathrm{LAI}=3.5)$.

\section{Results}

\subsection{Sensitivity study}

Figure 3 shows the success rate $S_{r}$ of retrieving component temperatures, for the sensors, temperature profiles and noise levels. The values of the component temperatures in most scenarios are not improved using the retrieval algorithm with monodirectional sensors and even deteriorate with increasing noise level:

1. The improvement of the retrieval depends on the number of unique measurements. The measurements per band are highly correlated (see Planck's function). Therefore no improvement is observed.

2. The retrieval algorithm only improves on the a priori information for temperature profile 1 , but the directional brightness temperature of $T$-profile 1 is does not exhibit directional behaviour. This directional brightness temperature is used as a priori information. As a priori temperature is based on brightness temperature, the retrieval algorithm improves upon first estimation this using the emissivities.

The values of the component temperatures in most scenarios are improved using retrieval algorithm with the multidirectional sensors. Although the success rate reduces with increasing noise levels, only for certain scenarios the success rate becomes unacceptable:
1. The improvement of the retrieval $\left(S_{r}<1.0\right)$ is apparent for all $T$-profiles with $N_{l}<1.0$. Even for very high noise levels $N_{l}=0.8$ the values of the success rate are below 1.0 .

2. The success rate for $\left(T\right.$-profile $\left.=1 @ N_{l}>1.0\right)$ is not acceptable. The retrieval of the component temperatures is actually worse than for monodirectional sensors. For $T$-profile 1 the directional brightness temperature is uniform over the viewing angles (Fig. 2). The shape of the added noise, controlled by $\Re$, will then dominate the shape of the directional brightness temperature. The retrieval algorithm tries to fit this shape, and therefore consequently will not find the correct canopy component temperatures. In reality the component temperatures will never have a complete homogeneous temperature.

3. For the scenario $\left(N_{l}=1.0\right)$ the success rate predominantly depends on the shape of the random values, $\Re$, which is unacceptable. Note that the added noise in this scenario is of the same order as the sensor sensitivity. This is an order of magnitude higher than usual sensor noise.

The best average success rate for $T$-profiles $2-7$ is achieved by multi-directional sensors: the SPECTRA sensor (0.3), than by the Goniometric setup (0.3) and the AATSR (0.5).

In addition to the retrieval of four parameters, two other inversions were performed to retrieve five and six parameters. The extra parameters to be retrieved were sky temperature 

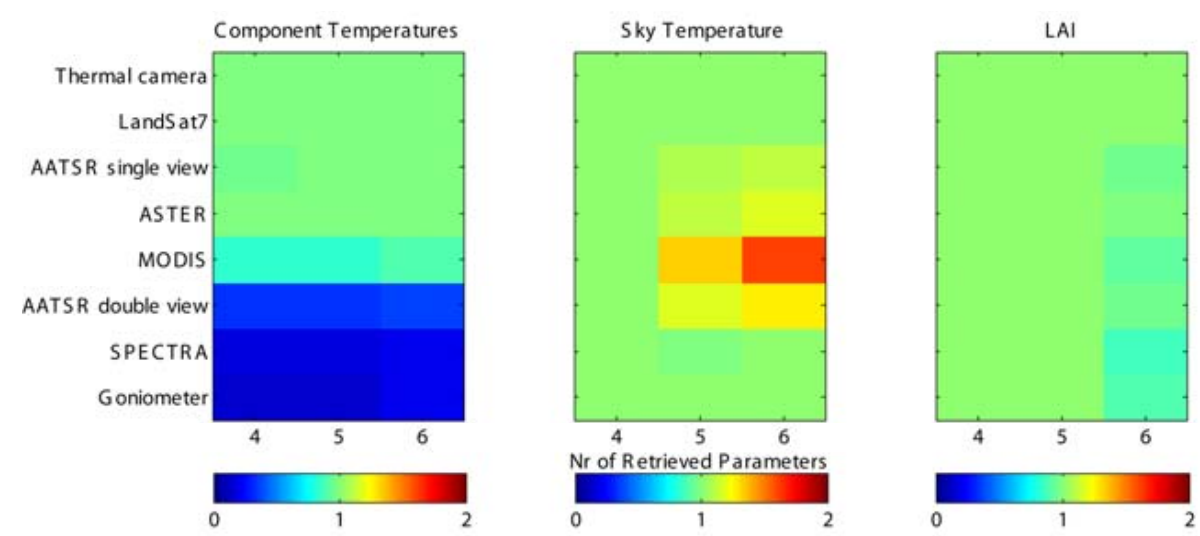

Fig. 4. Success rates on the retrieval of 4,5 and 6 parameters for the retrieval of Component temperatures, sky temperatures and LAI. In the case of 4 parameters, only component temperatures are retrieved, resulting in a success rate of 1 for sky temperature and LAI.

and leaf area index (LAI). The overall success rate of these runs is shown in Fig. 4.

1. The success rate for the component temperature remains acceptable, with only a slight reduction when retrieving six parameters.

2. The overall success rates for the retrieval of sky temperature are unacceptable $\left(S_{r}>1.0\right)$ for AATSR and MODIS. As the success rate of the component temperature is unaffected.

3. The overall success rates for the retrieval of LAI are promising $\left(S_{r} \leq 1.0\right)$. As LAI is usually retrieved using optical measurements this algorithm can be used to enhance this value with the extra information of thermal measurements.

\subsection{Field measurements retrieval}

Three time series were made with the goniometer during the EAGLE 2006 fieldcampaign. Of the three time series two were made over the tall grass and one time series was made over the maize (Fig. 5-6). The lines represent the mean measured brightness temperature. The error bars denote the RMSE between the measured and the retrieved directional brightness temperature. The RMSE for grass is about $0.5 \mathrm{~K}$ and for maize $2.5 \mathrm{~K}$. The difference between the measured and modelled will be explained in detail in the following section (discussion).

The measured brightness temperatures over the grass were performed during two days, 12 June 2006 and 14 June 2006. The brightness temperatures were higher on the first day than on the second day: on the first day the measurements were performed in the afternoon with clear skies, while on the second day the measurements were performed early in the morning with overcast conditions.

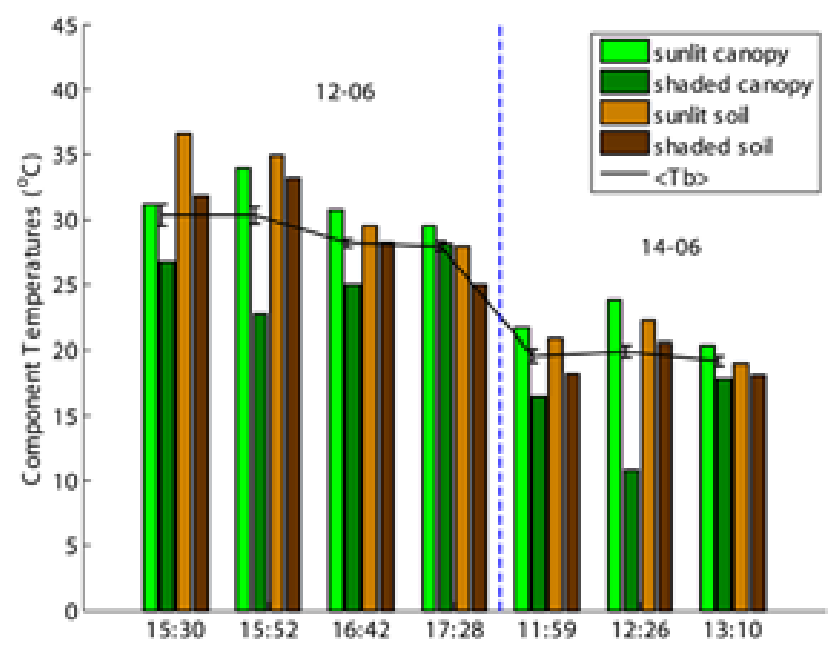

Fig. 5. Retrieved canopy component temperatures of grass on 12 June 2006 and 14 June 2006. The dots represent the mean directional brightness temperature; the error bars represent the RMSE between the measured en modelled brightness temperatures.

The grass temperatures at 15:30 and 15:52 on first day are lower than the soil temperatures. The soil temperature decreases during the day, where the vegetation temperature remains relatively constant. This is caused by the direct illumination sun on the different components. As the sun reaches lower angles, the soil is shaded by the canopy, whereas the leaves high in the canopy still receive sunlight.

The maize temperatures are always lower that the temperatures of soil. Both sunlit maize and soil display the same temporal behaviour as the directional brightness temperature. However shaded maize and soil do not display the same temporal behaviour. This is caused by the structural parameters of the maize. As the maize was very young the vegetation height was about $10 \mathrm{~cm}$ and the LAI about 0.2 . For high sun angles the thermal image was dominated by the sunlit soil, 


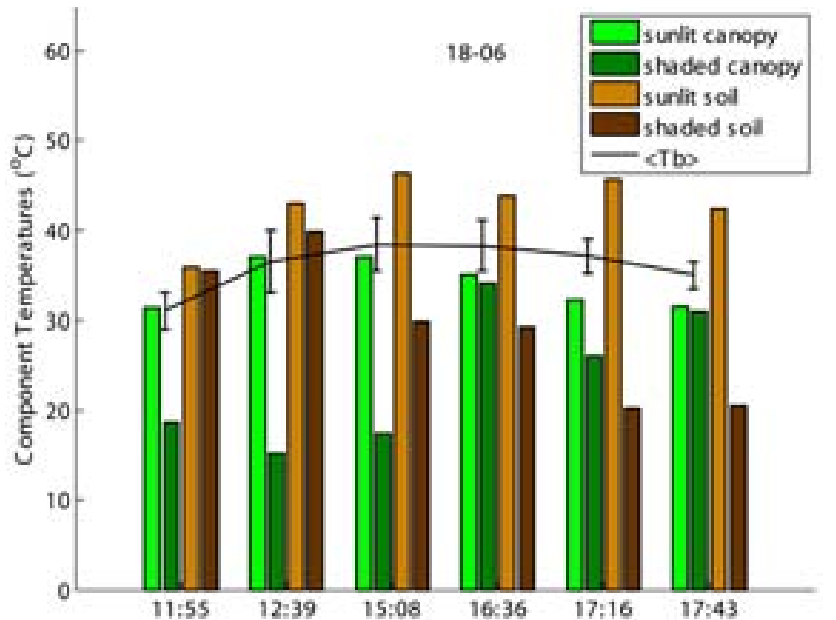

Fig. 6. Retrieved canopy component temperatures of young maize on 18 June 2006. The dots represent the mean directional brightness temperature; the error bars represent the RMSE between the measured en modelled brightness temperatures.

as the amount of shaded soil is very small due to the height of the canopy. For very low sun angles the thermal image is dominated by sunlit vegetation. The sensitivity of the shaded components is therefore very low for sparse low canopies.

During the AGRISAR campaign the emphasis of the measurements was on the effect of structural differences between crops on the directional brightness temperatures. Therefore the measurements were performed over more vegetation types, but with less temporal resolution (Fig. 7). The lines represent the mean measured brightness temperature. The error bars denote the RMSE between the measured and the retrieved directional brightness temperature. The RMSE for maize, wheat, sugar beet and barley is respectively $2.85 \mathrm{~K}$, $0.29 \mathrm{~K}, 0.75 \mathrm{~K}$ and $0.67 \mathrm{~K}$. The measurements of the maize are left out for further investigation because of the high RMSE for maize, and the absence of more (temporal) measurements

The variance in the observed component temperature differs between the crops. The largest variance between the component temperatures is found for sugar beet. Wheat and barley display similar behaviour. Both wheat and barley are considered very homogeneous as they were fully developed, have a large LAI, with similar leaves in terms of leaf angles scattering and absorption. This high level of homogeneity causes the relatively small variations in the vegetation temperatures. As the measurements were performed during midday the soil temperature are higher than the shaded vegetation temperatures.

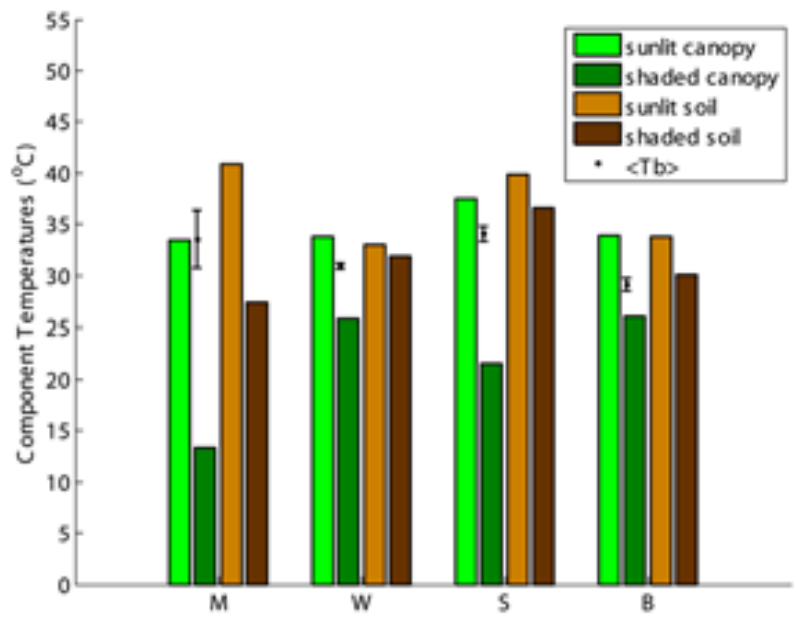

Fig. 7. The retrieved canopy component temperatures of maize (M), wheat (W), sugar beet (S) and barley (B). The dots represent the mean directional brightness temperature; the error bars represent the RMSE between the measured en modelled brightness temperatures.

\subsection{Discussion}

The potential of the Bayesian inversion and multidirectional imagery has been demonstrated. For monodirectional sensors no improvement upon the a priori estimates is found. The success rates values for monodirectional sensors are for the most part 1 . Only for unrealistically high noise profiles are there unacceptable found. As success rates of 1 denote no deterioration upon a priori estimates the algorithm can be run as a post-processor on Level 3 satellite imagery without cause for concern for deterioration of the products. The retrieval of component temperatures by forward simulated thermal (directional) brightness temperatures is successful for all the directional sensors, as the values for the success rate all pre-dominantly below 1 .

Of all possible view angles, the along-track configuration proves the highest potential. The SPECTRA and goniometric configuration have the same success rate. The additional cross solar plane angles therefore do not contribute a lot. Also in space cross solar planes viewing angles are impossible to retrieve. In the field a goniometric setup is preferred. With a SPECTRA configuration the setup should be changed every acquisition due the changing solar azimuth angles.

The retrieval of parameters other than the component temperatures by forward simulated thermal (directional) brightness temperatures is not successful for sky temperature and moderately successful for LAI. The sensitivity of directional radiation for sky temperature is very low. As a result the algorithm fails to retrieve this parameter. The sensitivity of directional radiation is very high for LAI, as is also proven in the AGRISAR field measurements. As LAI is usually retrieved using optical measurements the need for retrieval of this parameter through thermal measurements is low. How- 
ever in the absence of optical measurements, for example in night time, this procedure could be used.

The retrieval of component temperatures by measured thermal directional brightness temperatures is successful for all crops except mature maize. The retrieval resulted in a large RMSE between the retrieved and measured directional brightness temperature.

The values of the RMSE between retrieved and directional brightness temperature is influenced by the goniometric setup. In some of the thermal images the aluminum tracks of the goniometer are visible, and contaminate the measurement. These tracks have a significant lower brightness temperature than the vegetation or soil. The amount of track visible is much higher for low LAI with low vegetation height than for high LAI with high vegetation height. In the case of the mature maize (AGRISAR) even though the canopy had a high LAI and vegetation height, a gap in the canopy caused the visibility of the tracks. As these tracks usually have a significant temperature than the soil or canopy, the images can be filtered. As this is beyond the scope of this paper has not been considered.

\section{Conclusions}

A method is presented to retrieve up to four canopy component temperatures from directional measurements. The method inverts the radiative transfer model 4SAIL to retrieve the four component temperatures (sunlit/shaded vegetation/soil). The inversion is based on a modified GaussNewton optimization scheme. The modification incorporated the use of a priori knowledge to ensure closure of the inversion problem. Further measures, to ensure closure, were the introduction of singular value decomposition on the Jacobian sensitivity matrix.

The method was tested over a large dataset of forwardsimulated data, as a sensitivity analysis. The forward simulations were performed for 8 satellite sensors, 10 solar zenith angles 8 sensors and 6 noise levels. The solar zenith angles varied between 0 and 60 degrees. The temperature profiles were representative of component temperatures in the summer, winter, and autumn. One additional scenario (with homogeneous temperatures) was used, in order to test the system. The sensors that were simulated consisted of ground sensors, existing satellite sensors and future satellite sensors. The sensor characteristics like accuracy were used to normalize the parameters in the inversion scheme. The noise added to the forward simulations is also based on these sensor characteristics. Instead of the NEdT, the noise added to the (directional) brightness temperatures were a percentage of the sensor accuracy. Three retrieval runs were performed with four, five and six number of parameters to be retrieved, (four canopy components, sky temperature, and leaf area index).
The results of these sensitivity analyses prove that the algorithm is capable of using the extra information in the directional brightness temperature to retrieve the four component temperatures, and to lesser extent leaf area index. The retrieval of sky temperature fails with this method. Only an improvement on the a priori information was observed when using multidirectional imagery. Here the success rate of the inversion is good for all solar zenith angles, most temperature profiles and all but the highest noise levels. When the temperature profile is completely homogeneous, the success rate was unsatisfactory. For homogeneous temperature profiles the added noise introduces a shape in the "homogeneous" brightness temperature. The retrieval algorithm will then try to model this shape resulting in a worse estimation of the component temperatures. Fortunately this scenario is rarely observed, as sunlit components will almost always have a higher temperature than shaded components. In the case of cloudy days this scenario may occur, however the satellite will not be able to observe it.

The method was finally run over the datasets from two field campaigns. These datasets included a temporal datasets over grass and maize, and a multi-crop dataset over maize, wheat, sugar beet and barley. The method produced good results for the component temperature. However the RMSE between the measured and retrieved directional brightness temperature exceeded sensor accuracy levels for certain scenarios. This is caused by the aluminum track of goniometric setup that has a different temperature than the four canopy components. As these temperatures are not filtered out of the thermal images, they produce an RMSE of between $0.5 \mathrm{~K}$ and $2.5 \mathrm{~K}$, depending on the LAI. As these errors were not present in the sensitivity analysis, which was performed with a low LAI, we are confident that filtering the images for these tracks will increase the efficiency of the inversion method.

Acknowledgements. This research was performed under the framework of "Modeling radiation, heat and mass (water vapor and carbon) exchanges at the land-atmosphere interface using multi-angular optical and thermal measurements", project number EO-071. This project was funded by the Netherlands Institute for Space Research (SRON). The EAGLE and AGRISAR campaigns were carried out in the framework of the Earth Observation Envelope Program of the ESA, and were partly financed by the EU 6FP Project, SRON EO-071, and the ITC International Institute for Geo-Information Science and Earth Observation. The authors thank, Zoltan Vekerdy, Kitsiri Weligepolage, Wim Timmermans and Remco Dost for fieldwork and data processing and coordination during the EAGLE and AGRISAR campaigns.

Edited by: J. Wen 


\section{References}

Bastiaanssen, W. G. M., Menenti, M., Feddes, R. A., and Holtslag, A. A. M.: a Remote Sensing Surface Energy Balance Algorithm, for land (SEBAL) 1: Formulation, J. Hydrol., 212-213, 198212, 1998.

D‘Urso, G., Dini, L., Vuolo, F., Alonso, L., and Guanter, L.: Retrieval of Leaf Area Index by Invering Hyper-spectral MultiAngular CHRIS/PROBA data from SPARC 2003, Procedures of the 2nd CHRIS/PROBA Workshop, ESA SP-578, ESA/ESRIN, Frascati, 2004.

Fox, J.: Applied regression analysis, linear models and related methods, Sage Publications, 2455 Teller Road, Thousand Oaks, California, p. 597, 1997.

Gastellu-Etchegorry, J. P., Gascon, F., and Estève, P.: An interpolation procedure for generalizing a look-up table inversion method, Remote Sens. Environ., 87, 55-71, 2003.

Gastellu-Etchegorry, J. P., Martin, E., and Gascon, F.: DART: a 3D model for simulating satellite images and studying surface radiation budget, 2004, Int. J. Remote Sens., 25(1), 73-96, 2004.

Gillespie, A., Rokugawa, S., Matsunaga, T., Cothern, J. S., Hook, S., and Kahle, A. B.: A Temperature and Emissivity Separation Algorithm for Advanced Spaceborne Thermal Emission and Reflectance Radiometer (ASTER) Images, IEEE T. Geosci. Remote, 36(4), 1113-1226, 1998.

Jacquemoud, S. and Baret, F.: PROSPECT: a model of leaf optical properties spectra, Remote Sens. Environ., 34, 75-91, 1990.

Jia, L., Su, Z., van den Hurk, B., Menenti, M., Moene, A., de Bruin, H. A. R., Baselga Yrisarry, J. J. B., Ibanez, M., and Cuesta, A.: Estimation of Sensible Heat Flux using the Surface Energy Balance System (SEBS) and ATSR Measurements, Phys. Chem. Earth, 28, 75-88, 2003a.

Jia, L., Li, Z.-1., Menenti, M., Su, Z., Verhoef, W., and Wan, Z.: A practical algorithm to infer soil and foliage component temperatures from bi-angular ATSR-2 data, Int. J. Remote Sens., 24(23), 4739-4760, 2003b.

Kimes, D. S., Knyazikhin, Y., Privette, J. L., AbuelGasim, A. A., and Gao, F.: Inversion Methods for Physically-Based Models, Remote Sens. Rev., 18, 381-439, 2000.

Knyazikhin, Y., Martonchik, J. V., Diner, D. J., Myneni, R. B., Verstraete, M., Pinty, B., and Gobron, N.: Estimation of Vegetation Canopy Leaf Area Index and Fraction of Absorbed Photosynthetically active Radiation from Atmosphere-corrected MISR data, J. Geophys. Res., 103(D24), 32239-32256, 1998.

Kustas, W. P. and Norman, J. M.: A two-Source Energy Balance Approach using Directional Radiometric Temperature Observations for Sparse Canopy Covered Surfaces, Agron. J., 92, 847$854,2000$.

Li, Z.-1., Zhang, R., Sun, X., Su, H., Tang, X., Zhu, Z., and Sobrino, J. A.: Experimental system for the study of the directional thermal emission of natural surfaces, Int. J. Remote Sens., 25(1), 245-259, 2004.

Martonchik, J. V., Diner, D. J., Pinty, B., Verstraete, M. M., Myeni, R. B., Knyazikhin, Y., and Gordon, H. R.: Determination of Land and Ocean Reflective, Radiative, and Biophysical Properties using Multiangle Imagery, IEEE T. Geosci. Remote, 36(4), 12661281, 1998.

Moré, J. J. and Wright, S. J.: Optimization software guide, Frontiers in Applied Mathematics series, Vol. 14., Society for Industrial and Applied Mathematics (SIAM), Philadelphia, 1993.
Norman, J. M., Kustas, W. P., and Humes, K. S.: Source Approach for estimating soil and vegetation energy fluxes in observations of directional radiometric surface temperature, Agr. Forest Meteorol., 77, 263-293, 2005.

North, P. R. J.: Estimation of Aerosol opacity and land surface bidirectional reflectance from ATSR-2 dual angle imagery: operational method and validation, J. Geophys. Res., 107(D12), 4149, doi:10.1029/2000JD000207, 2002.

Payan, V. and Royer, A.: Analysis of Temperature Emissivity Separation (TES) algorithm applicability and sensitivity, Int. J. Remote Sens., 25(1), 15-37, 2004.

Picton, P.: Neural Networks, $2^{\text {nd }}$ edition, Palgrave, Grassroot series 2000, Basingstoke New York, 0-333-80287-X, 195 pp., 2000.

Pinty, B., Gobron, N., Widlowski, J.-L., Gerstl, S. A. W., Verstraete, M. M., Antunes, M., Bacour, C., Gascon, F., Gastellu, J.-P., Goel, N., Jacquemoud, S., North, P., Qin, W., and Thompson, R.: The Radiation transfer Model (RAMI) Exercise, J. Geophys. Res. IWMMM-2 Special issue, 106, 11937-11956, 2001.

Pinty, B., Widlowski, J.-L., Taberner, M., Gobron, N., Verstraete, M. M., Disney, M., Gascon, F., Gastellu, J.-P., Jiang, L., Kuusk, A., Lewis, P., Li, X., Ni-Meister, W., Nilson, T., North, P., Qin, W., Su, L., Tang, S., Thompson, R., Verhoef, W., Wang, H., Wang, J., Yan, G., and Zang, H.: Radiation Transfer Model Intercomparison (RAMI) exercise: Results from the second phase, J. Geophys. Res., 109, D06210, doi:10.1029/2003JD004252, 2004.

Prata, A. J. F., Cechet, R. P., Barton, I. J., and Llewellyn-Jones, D. T.: The Along Track Scanning Radiometer for ERS-1-Scan Geometry and Data Simulation, IEEE T. Geosci. Remote, 28(1), 3-13, 1990.

Press, W. H., Flannery, B. P., Teukolsky, S. A., and Vetterling, W. T.: Numerical Recipes in C: the art of scientific computing, Third Edition, 1256 pp., Cambridge University Press ISBN-10: 0521880688, 2007.

Quattrochi, D. A. and Luvall, J. C.: Thermal Remote Sensing in Land Surface Processes, CRC Press, Albert House, 4th floor 1-4 Singer Street London EC2A 4BQ, United Kingdom, 2004.

Sobrino, J. A. and Jiménez-Munõz, J. C.: Land surface temperature Retrieval from Thermal Infrared data: An assessment in the context of the Surface Process and Ecosystem Changes through Response Analysis (SPECTRA) mission, J. Geophys. Res., 110, D16103, doi:10.1029/2004JD005588, 2005.

Sobrino, J. A., Romaguera, M., Sòria, G., Zaragoza, M. M., Gomez, M., Cuenca, J., Julien, Y., Jiménez-Muñoz, J. C., Su, Z., Jia, L., Gieske, A. S. M., Timmermans, W. J., van der Kwast, H., Olioso, A., Nerry, F., Sabol, D., and Moreno, J.: Thermal measurements in the framework of SPARC, in: Proceedings of the ESA WPP250: SPARC final workshop, 4-5 July, 2005, 6 pp., Enschede: ESA, 2005.

Su, Z.: The Surface Energy Balance System (SEBS) for estimation of turbulent heat fluxes, Hydrol. Earth Syst. Sc., 6(1), 85-99, 2002.

Su, Z., Timmermans, W. J., Dost, R., Bianchi, R., Gómez, J. A., House, A., Hajnsek, I., Menenti, M., Magliulo, V., Esposito, M., Haarbrink, R., Bosveld, F., Rothe, R., Baltink, H. K., Vekerdy, Z., Sobrino, J. A., van der Tol, C., Timmermans, J., van Laake, P., Salama, S., van der Kwast, H., Claassen, E., Stolk, A., Jia, L., Moors , E., Hartogensis, O., and Gillespie, A.: EAGLE 2006 - Multi-purpose, multi-angle and multi-sensor in-situ and airborne campaigns over grassland and forest, in: Proceed- 
ings of the AGRISAR and EAGLE campaigns, final workshop, ESA/ESTEC, Noordwijk, The Netherlands, ESA, 2007 (ESA Proceedings WPP-279), 11 pp., 2007.

Su, Z., Timmermans, W. J., van der Tol, C., Dost, R., Bianchi, R., Gómez, J. A., House, A., Hajnsek, I., Menenti, M., Magliulo, V., Esposito, M., Haarbrink, R., Bosveld, F., Rothe, R., Baltink, H. K., Vekerdy, Z., Sobrino, J. A., Timmermans, J., van Laake, P., Salama, S., van der Kwast, H., Claassen, E., Stolk, A., Jia, L., Moors, E., Hartogensis, O., and Gillespie, A.: EAGLE 2006 Multi-purpose, multi-angle and multi-sensor in-situ and airborne campaigns over grassland and forest, Hydrol. Earth Syst. Sci., 13, 833-845, 2009, http://www.hydrol-earth-syst-sci.net/13/833/2009/.

Tarantola, A.: Inverse Problem Theory and Methods for Model Parameter Estimation, SIAM: Society for Industrial and Applied Mathematics. ISBN-10: 0898715725, ISBN-13: 9780898715729, 2005.

Timmermans, J., van der Tol, C., Verhoef, W., and Su, Z.: Contact and directional radiative temperature measurements of sunlit and shaded land surface components during the SEN2FLEX 2005 campaign, Int. J. Remote Sens., 29,(17), 5183-5192, 2008.

Timmermans, J., Gieske, A. S. M., van der Tol, C., Verhoef, W., and $\mathrm{Su}, \mathrm{Z}$.: Automated directional measurement system for the acquisition of thermal radiative measurements of vegetative canopies, Sensors, 9, 1409-1422, doi:10.3390/s90301409, 2009.

Timmermans, W. J., van der Kwast, H., Gieske, A. S. M., Su, Z., Olioso, A., Jia, L., and Elbers, J.: Intercomparison of energy flux models using ASTER imagery at the SPARC 2004 site (Barrax, Spain), in: Proceedings of the ESA WPP-250: SPARC final workshop, 8 pp., 4-5 July, 2005.

van der Tol, C., Verhoef, W., Timmermans, J., Verhoef, A., and Su, Z.: An integrated model of soil-canopy spectral radiance observations, photosynthesis, fluorescence, temperature and energy balance, Biogeosciences Discuss., 6, 6025-6075, 2009, http://www.biogeosciences-discuss.net/6/6025/2009/.

Van Ulden, A. P. and Wieringa, J.: Atmospheric Boundary Layer Research at Cabauw, Bound.-Lay. Meteorol., 78, 39-69, 1996.
Verhoef, W.: Light Scattering by Leaf Layers with Application to Canopy Reflectance Modeling: The SAIL model, Remote Sens. Environ., 16, 124-141, 1984.

Verhoef, W.: Simultaneous retrieval of soil, leaf, canopy and atmospheric parameters from hyperspectral information in the red edge through model inversion, report for National Aerospace Laboratory NLR, 20, 2000.

Verhoef, W., Jia, L., Xiao, Q., and Su, Z.: Unified optical - thermal four - stream radiative transfer theory for homogeneous vegetation canopies, IEEE T. Geosci. Remote, 45(6), 1808-1822, 2007a.

Verhoef, W.: A Bayesian Optimizations approach for Model Inversion of Hyperspectral-Multidirectional Observations: The Balance with a priori Information, proc. of the 10th Int. Symposium on Phys. Measurements and Spectral Signatures in Remote Sensing (ISPMSRS'07), Davos, Switzerland, 208-213, March 2007b.

Widlowski, J.-L., Taberner, M., Pinty, B., Bruniquel-Pinel, V., Disney, M., Fernandes, R., Gastellu-Etchegorry, J.-P., Gobron, N., Kuusk, A., Lavergne, T., Leblanc, S., Lewis, P. E., Martin, E., Mõttus, M., North, P. R. J., Qin, W., Robustelli, M., Rochdi, N., Ruiloba, R., Soler, C., Thompson, R., Verhoef, W., Verstraete, M. M., and Xie, D.: Third radiation transfer model intercomparison (RAMI) exercise: documenting progress in canopy reflectance models, J. Geophys. Res., 112(D9), D09111, ISSN 01480227, 2007.

Yamaguchi, Y., Kahle, A. B., Tsu, H., Kawakami, T., and Pniel, M.: Overview of Advanced Spaceborne Thermal Emission and Reflectance Radiometer (ASTER), IEEE T. Geosci. Remote, 36(4), 1062-1071, 1998.

Yan, G., Friedl, M., Li, X., Wang, J., Zhu, C., and Strahler, A. H.: Modeling Directional Effects from Nonisothermal Land Surfaces in Wideband Thermal Infrared Measurements, IEEE T. Geosci. Remote, 39(5), 1095-1099, 2001. 